\title{
A Novel Entry into Cyclopropanated Sugar Amino Acids
}

\author{
Fakhar-uz-Zaman, Ayjaz Fatima, Abdul Malik, Wolfgang Voelter* \\ Abteilung für Physikalische Biochemie des Physiologisch-chemischen Instituts der \\ Universität Tübingen, Hoppe-Seyler-Straße 4, D-72076 Tübingen, Germany \\ Z. Naturforsch. 49b, 1434-1438 (1994); received May 16, 1994 \\ Anhydro Sugars, Sugar Triflates, Cyclopropanated Sugar Amino Acids
}

Reaction of sugar triflates (1) and (2) with tert-butyl cyanoacetate in presence of sodium hydride affords the cyclopropanated sugars (3) and (4), followed by selective hydrolysis of the ester group to free acids (5) and (6), respectively. Couplings of (5) and (6) with protected glycine and L-alanine lead to cyclopropanated sugar amino acids (7-10). The coupling of 6 with benzyl 3,4-("exo"-aminomethyl)methano-3,4-dideoxy- $\beta$-L-arabinopyranoside (11) furnished benzyl 3,4-[(C-cyano-amido)methano-(benzyl 3,4-("exo"-methylene)methano-3,4-dideoxy- $\beta$-L-arabinopyranosido)]-3,4-dideoxy- $\beta$-L-arbinopyranoside (12), suggesting an "exo" orientation of the ester group in $\mathbf{3}$ and $\mathbf{4}$.

\section{Introduction}

A recent report from this laboratory [1] described a simple sequence of reactions for converting the readily available L-arabinose and Darabinose into 3,4-cyclopropanated sugars via 2,3anhydropyranosides. The route has considerable potential for syntheses of structural variants of 3,4cyclopropanated sugars by appropriate modification of steps from the key intermediates. The present work describes such a variation to afford high-yielding syntheses of a new type of cyclopropanated sugar amino acids of potential biochemical interest.

\section{Results and Discussion}

The benzyl 2,3-anhydro-4-O-trifluoromethanesulfonyl- $\beta$-L-ribopyranoside (1) and its $\alpha$-D-isomer (2) are prepared by previously published methods [2-4] from L- and D-arabinose, respectively, and both of these are treated with tert-butyl cyanoacetate in the presence of sodium hydride to obtain the cyclopropanated sugar derivatives (3) and (4). Apparently, the sodium enolate of tertbutyl cyanoacetate initially displaces, with inversion, the triflyl group which is followed by further alkylation by the trans-oriented oxirane ring. The two different conformers ${ }^{1} \mathrm{H}_{\mathrm{o}}$ and ${ }^{\circ} \mathrm{H}_{1}$ can be safely assigned to the compounds $\mathbf{3}$ and $\mathbf{4}$, based

\footnotetext{
* Reprint requests to Prof. Dr. Dr. h.c. Wolfgang Voelter.
}

on the chemical shifts of $1-\mathrm{H}$ in their ${ }^{1} \mathrm{H}$ NMR spectra [1]. Interestingly, we obtained a complete control on the newly formed chiral center, i.e. the bulky ester group is apparently in "exo" orientation in both the cases, as suggested by the proceeding reactions. The selective hydrolysis of the ester function with normal acidic and basic reagents caused considerable difficulties due, either to the hydrolysis of both the cyano and ester moieties, or formation of a complex mixture of products. However, saponification with $80 \%$ trifluoroacetic acid in dichloromethane cleanly affords the free acids (5) and (6) in almost theoretical yields. The compound $\mathbf{5}$ is reacted with hydrochlorides of carboxyl- protected naturally occurring amino acids including methyl- and benzyl- esters of glycine and the benzyl ester of L-alanine, respectively. The reactions are carried out in dimethylformamide in the presence of 4-methylmorpholine, affecting coupling with "Castro Reagent" $\left(\mathrm{BOP}^{+} \mathrm{PF}_{6}^{-}\right)[5-6]$ to yield cyclopropanated sugar amino acids (7-9). The analogous reaction of 6 with the benzyl ester of glycine leads to compound 10. The compounds $\mathbf{5 , 7 , 8}$ and 9 are shown to exist almost entirely in the ${ }^{1} \mathrm{H}_{\mathrm{o}}$ conformation by ${ }^{1} \mathrm{H}$ NMR spectroscopy, while ${ }^{\circ} \mathrm{H}_{1}$ can be assigned to 6 and 10. Similar conformations are observed for $\mathbf{3}$ and $\mathbf{4}$ from which it can be inferred that hydrolysis of the ester group and subsequent amide bond formation does not change the conformational stability of the molecule (Scheme 1).

The bulky ester group in compounds $\mathbf{3}$ and $\mathbf{4}$ was expected to be in "exo" orientation according 

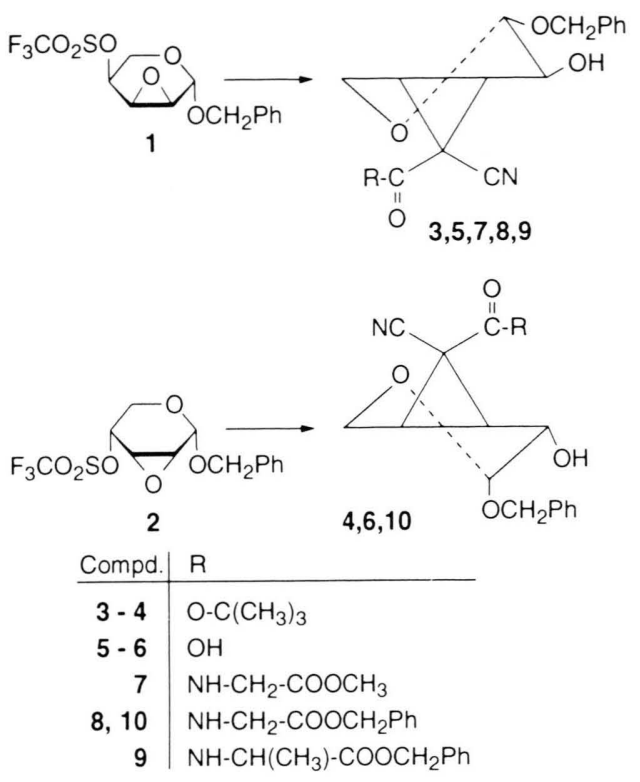

Scheme 1.

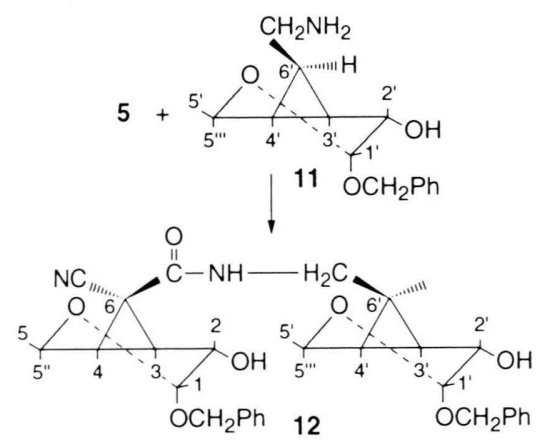

Scheme 2 .

to earlier findings of Schöllkopf et al. [7-8] in analogous but simpler cyclopropane derivatives. Further indication to this effect is provided by coupling of 6 with benzyl 3,4-("exo"-aminomethyl)methano-3,4-dideoxy- $\beta$-L-arabinopyranoside (11) [1] under the conditions described in the foregoing. The facile coupling and high yield of the resulting compound $\mathbf{1 2}$ suggest similar orientation of the carboxyl group in $\mathbf{5}$ (and hence the ester moiety in 3) as that of the aminomethyl group in $\mathbf{1 1}$ for which "exo"-orientation has already been established [1]. The evidence, however, is not con- clusive and further insight to the stereochemistry of the newly forming chiral centre in 6,6- disubstituted-3,4-cyclopropanated sugars would be provided later with the help of X-ray crystallography. The ${ }^{1} \mathrm{H}$ NMR spectrum shows that ${ }^{\circ} \mathrm{H}_{1}$ conformations are retained by both the sugar molecules of product 12, once again revealing that amide bond formation does not change the conformational stability of this type of compounds (Scheme 2).

\section{Experimental}

The melting points are recorded on a Büchi 510 apparatus and are uncorrected. The optical rotations are measured on a digital polarimeter OLD 5, Carl Zeiss. The elemental analyses are performed on a Carlo Erba elemental analyzer. The field desorption mass spectra are recorded on a Varian MAT 711 mass spectrometer and the NMR spectra on a Bruker WM-400 spectrometer. The assignments of the ${ }^{13} \mathrm{C}$ NMR spectra are made through comparison with the corresponding ones of a similar series of compounds [1] as well as published data of various carbohydrate derivatives [9].

Benzyl 3,4-(C-cyano-tert-butyloxycarbonyl)methano-3,4-dideoxy- $\alpha-D$-arabinopyranoside (3): A solution of $0.1 \mathrm{~g}(3.33 \mathrm{mmol})$ sodium hydride in 10 $\mathrm{ml}$ abs. tetrahydrofuran is cooled to $0{ }^{\circ} \mathrm{C}$, then dropwise $0.5 \mathrm{ml}(3.58 \mathrm{mmol})$ tert-butyl cyanoacetate are added and stirred for $10 \mathrm{~min}$. A solution of $1 \mathrm{~g}(2.82 \mathrm{mmol})$ benzyl 2,3-anhydro-4-O-trifluoromethanesulfonyl- $\beta$-L-ribopyranoside (1) in 10 $\mathrm{ml}$ tetrahydrofurane is slowly added and stirred for further $20 \mathrm{~min}$ at $0^{\circ} \mathrm{C}$. Then the reaction mixture is kept at room temperature for $5 \mathrm{~h}$, the solvent removed, $15 \mathrm{ml}$ water added and extracted thrice with $20 \mathrm{ml}$ dichloromethane. The organic phase is washed with $50 \mathrm{ml}$ water, $30 \mathrm{ml}$ saturated sodium chloride solution, dried over $\mathrm{Na}_{2} \mathrm{SO}_{4}$, freed of the solvent and crystallized from ether $/ n$ hexane as colourless needles. Yield $0.82 \mathrm{~g}(84 \%)$, m.p. $156-157^{\circ} \mathrm{C},[\alpha]_{\mathrm{D}}=-25.5^{\circ}\left(\mathrm{c}=1, \mathrm{CHCl}_{3}\right)$. FAB-MS: $m / z=346(\mathrm{M}+1)^{+} .-{ }^{1} \mathrm{H}$ NMR $(400$ $\left.\mathrm{MHz}, \mathrm{CDCl}_{3}\right): \delta=7.36-7.27(\mathrm{~m}, 5 \mathrm{H}, \mathrm{Ar}-\mathrm{H}), 4.87$ $\left(\mathrm{d}, 1 \mathrm{H}, J_{\text {gem }}=11.6 \mathrm{~Hz}, \mathrm{OC} \underline{\mathrm{HPh}}\right), 4.51(\mathrm{~d}, 1 \mathrm{H}$, $\left.J_{\mathrm{gem}}=11.6 \mathrm{~Hz}, \mathrm{OCH} \mathrm{Hh}\right), 4.36\left(\mathrm{~d}, 1 \mathrm{H}, J_{5^{\prime} .5}=12.9\right.$ $\left.\mathrm{Hz}, 5^{\prime}-\mathrm{H}\right), 4.16\left(\mathrm{~d}, 1 \mathrm{H}, J_{1,2}=7.5 \mathrm{~Hz}, 1-\mathrm{H}\right), 4.14$ $\left(\mathrm{dd}, 1 \mathrm{H}, J_{5.4}=3.5 \mathrm{~Hz}, J_{5.5^{\prime}}=13.1 \mathrm{~Hz}, 5-\mathrm{H}\right), 3.84$ $\left(\mathrm{ddd}, 1 \mathrm{H}, J_{2,1}=7.4 \mathrm{~Hz}, J_{2, \mathrm{OH}}=2.9 \mathrm{~Hz}, J_{2,3}=1.2\right.$ $\mathrm{Hz}, 2-\mathrm{H}), 2.38\left(\mathrm{~d}, 1 \mathrm{H}, J_{\mathrm{OH}, 2}=2.9 \mathrm{~Hz}, \mathrm{OH}\right), 2.17$ $\left(\mathrm{dd}, 1 \mathrm{H}, J_{3,4}=9.5 \mathrm{~Hz}, J_{3,2}=1.1 \mathrm{~Hz}, 3-\mathrm{H}\right), 2.01(\mathrm{dd}$, $\left.1 \mathrm{H}, J_{4,3}=9.5 \mathrm{~Hz}, J_{4,5}=3.4 \mathrm{~Hz}, 4-\mathrm{H}\right), 1.48(\mathrm{~s}, 9 \mathrm{H}$, 
$\left.\mathrm{C}\left(\mathrm{CH}_{3}\right)_{3}\right) .-{ }^{13} \mathrm{C} \mathrm{NMR}\left(100 \mathrm{MHz}, \mathrm{CDCl}_{3}\right): \delta=$ $165.6(\mathrm{C}=\mathrm{O}), 137.0-128.0(\mathrm{Ar}-\mathrm{C}), 114.3(\mathrm{CN})$, $101.7(\mathrm{C}-1), 84.3\left(\mathrm{C}\left(\mathrm{CH}_{3}\right)_{3}\right), 70.3\left(\mathrm{O}-\mathrm{CH}_{2} \mathrm{Ph}\right)$, $66.0(\mathrm{C}-2), 62.0(\mathrm{C}-5), 32.4(\mathrm{C}-3), 27.9\left(\mathrm{CH}_{3}\right), 27.3$ (C-4), $23.1(\mathrm{C}-6)$.

\section{$\mathrm{C}_{19} \mathrm{H}_{23} \mathrm{NO}_{5}(345.39)$ \\ Calcd C 66.07 H 6.71 N $4.05 \%$, \\ Found C 66.75 H 6.62 N $4.06 \%$.}

Benzyl 3,4-C-(cyano-tert-butloxycarbonyl)methano-3,4-dideoxy- $\beta$ - $L$-arabinopyranoside (4): Is prepared from $\mathbf{2}$ in exactly the same manner as described for 3. Colourless needles from ether $/ n$ hexane. Yield $0.85 \mathrm{~g}(87 \%)$, m.p. $111-112{ }^{\circ} \mathrm{C}$, $[\alpha]_{\mathrm{D}}=+131.2^{\circ}\left(\mathrm{c}=1, \mathrm{CHCl}_{3}\right) .-$ FD-MS: $m / z 346$ $(\mathrm{M}+1)^{+} .-{ }^{1} \mathrm{H}$ NMR $\left(400 \mathrm{MHz}, \mathrm{CDCl}_{3}\right): \delta=7.38-$ $7.32(\mathrm{~m}, 5 \mathrm{H}, \mathrm{Ar}-\mathrm{H}), 4.85\left(\mathrm{~d}, 1 \mathrm{H}, J_{1,2}=4.6 \mathrm{~Hz}, 1-\right.$ $\mathrm{H}), 4.77\left(\mathrm{~d}, 1 \mathrm{H}, J_{\mathrm{gem}}=11.6 \mathrm{~Hz}, \mathrm{OC} \underline{\mathrm{HPh}}\right), 4.54(\mathrm{~d}$, $\left.1 \mathrm{H}, J_{\mathrm{gem}}=11.6 \mathrm{~Hz}, \mathrm{OC} \underline{\mathrm{HPh}}\right), 4.07\left(\overline{\mathrm{dd}}, 1 \mathrm{H}, J_{5^{\prime}, 4}=\right.$ $\left.3.7 \mathrm{~Hz}, J_{5^{\prime}, 5}=12.8 \mathrm{~Hz}, 5^{\prime}-\mathrm{H}\right), 3.95\left(\mathrm{~d}, 1 \mathrm{H}, J_{5,5^{\prime}}=\right.$ $12.8 \mathrm{~Hz}, 5-\mathrm{H}), 3.86\left(\mathrm{dd}, 1 \mathrm{H}, J_{2,1}=4.6 \mathrm{~Hz}, J_{2, \mathrm{OH}}=\right.$ $11.0 \mathrm{~Hz}, 2-\mathrm{H}), 2.63\left(\mathrm{~d}, 1 \mathrm{H}, J_{\mathrm{OH}, 2}=11.0 \mathrm{~Hz}, \mathrm{OH}\right)$, $2.15\left(\mathrm{~d}, 1 \mathrm{H}, J_{3.4}=10.0 \mathrm{~Hz}, 3-\mathrm{H}\right), 2.08(\mathrm{dd}, 1 \mathrm{H}$, $\left.J_{4,3}=9.9 \mathrm{~Hz}, J_{4,5^{\prime}}=3.7 \mathrm{~Hz}, 4-\mathrm{H}\right), 1.47(\mathrm{~s}, 9 \mathrm{H}$, $\left.\mathrm{C}\left(\mathrm{CH}_{3}\right)_{3}\right) .-{ }^{13} \mathrm{C}$ NMR $\left(100 \mathrm{MHz}, \mathrm{CDCl}_{3}\right): \delta=$ 165.7 (CO), 136.3-128.2 (Ar-C), $114.4(\mathrm{CN}), 93.0$ $(\mathrm{C}-1), 84.2\left(\underline{\mathrm{C}}\left(\mathrm{CH}_{3}\right)_{3}, 70.2\left(\mathrm{OCH}_{2}-\mathrm{PH}\right), 62.1\right.$ (C-2), 54.2 (C-5), 31.1 (C-3), $27.8\left(\mathrm{CH}_{3}\right), 26.8$ (C-4), 23.1 (C-6).

\section{$\mathrm{C}_{19} \mathrm{H}_{23} \mathrm{O}_{5} \mathrm{~N}(345.39)$ \\ Calcd C 66.07 H 6.71 N 4.05\%, \\ Found C 65.75 H 6.62 N 4.22\%.}

Benzyl 3,4-(C-cyano-carboxy)methano-3,4-dideoxy- $\alpha$-D-arabinopyranoside $(\mathbf{5}): 1 \mathrm{~g}(2.89 \mathrm{mmol}) \mathbf{3}$ is stirred with $30 \mathrm{ml}$ of an $80 \%$ cooled solution of trifluoroacetic acid in dichloromethane for $30 \mathrm{~min}$. The solvent removed in vacuo and the residue dried under high vacuum over phosphorous pentoxide. Yield $0.82 \mathrm{~g}(98 \%)$, m.p. $58-60{ }^{\circ} \mathrm{C},[\alpha]_{\mathrm{D}}=$ $-21.3^{\circ}\left(\mathrm{c}=1, \mathrm{CHCl}_{3}\right)$. - FD-MS: $m / z 290(\mathrm{M}+1)^{+}$. The ${ }^{1} \mathrm{H}$ and ${ }^{13} \mathrm{C}$ NMR spectra are similar to $\mathbf{3}$ except the disappearance of peaks due to the methyl resonances of the tert-butyl ester moiety.

$$
\begin{array}{cccc}
\mathrm{C}_{15} \mathrm{H}_{15} \mathrm{NO}_{5} & \multicolumn{2}{c}{(289.28)} \\
\text { Calcd } & \text { C } 62.28 & \text { H } 5.22 & \text { N } 4.84 \%, \\
\text { Found } & \text { C } 62.01 & \text { H } 5.53 & \text { N } 4.36 \% .
\end{array}
$$

Benzyl 3,4-(C-cyano-carboxy)methano-3,4-dideoxy- $\beta$-L-arabinopyranoside (6): Is obtained from 4 in the same manner as 5. Yield $0.73 \mathrm{~g} \mathrm{(97 \% ),} \mathrm{m.p.}$ $52-54{ }^{\circ} \mathrm{C},[\alpha]_{\mathrm{D}}=11.9^{\circ}\left(\mathrm{c}=1, \mathrm{CHCl}_{3}\right)$. - FD-MS: $m / z 290(\mathrm{M}+1)^{+}$. The ${ }^{1} \mathrm{H}$ and ${ }^{13} \mathrm{C}$ NMR spectra are similar to 4 except the disappearance of the methyl peaks of the tert-butyl ester group.

$$
\begin{array}{rrrr}
\mathrm{C}_{15} \mathrm{H}_{15} \mathrm{NO}_{5}(289.28) & & \\
\text { Calcd } & \mathrm{C} 62.28 & \mathrm{H} 5.23 & \text { N } 4.84 \%, \\
\text { Found } & \text { C } 61.87 & \text { H } 5.35 & \text { N } 4.75 \% .
\end{array}
$$

Benzyl 3,4-(C-cyano-methyl glycinato)methano3,4-dideoxy- $\alpha$-D-arabinopyranoside (7): $0.35 \mathrm{~g}$ $(1.20 \mathrm{mmol})$ of 5 and $0.17 \mathrm{~g}(1.35 \mathrm{mmol})$ of the hydrochloride of methyl glycinate are dissolved in $20 \mathrm{ml}$ dimethylformamide and cooled at $0{ }^{\circ} \mathrm{C}$ for $20 \mathrm{~min}$. Afterwards $0.3 \mathrm{ml}(2.72 \mathrm{mmol})$ 4-methylmorpholine and $1.07 \mathrm{~g}(2.41 \mathrm{mmol}) \mathrm{BOP}$ are added. After $6 \mathrm{~h}$ the solvent is removed in vacuo and the residue taken up in $50 \mathrm{ml}$ ethyl acetate. After washing 3 times with a $10 \%$ solution of sodium bicarbonate, water, $10 \%$ dil. hydrochloric acid, water, drying over anhydrous $\mathrm{Na}_{2} \mathrm{SO}_{4}$ and removal of the solvent, an oily residue is obtained which is column-chromatographed over silica gel using methanol-ethyl acetate $(1: 4)$ as eluent. The product crystallizes from ethyl acetate/petroleum ether as colourless needles. Yield $0.38 \mathrm{~g}$ (87\%), m.p. $50{ }^{\circ} \mathrm{C},[\alpha]_{\mathrm{D}}=-15.4^{\circ}\left(\mathrm{c}=1, \mathrm{CHCl}_{3}\right)$. - FD-MS: $m / z=361(\mathrm{M}+1)^{+} .-{ }^{1} \mathrm{H}$ NMR $\left(400 \mathrm{MHz}, \mathrm{CDCl}_{3}\right)$ : $\delta=7.32-7.26(\mathrm{~m}, 5 \mathrm{H}, \mathrm{Ar}-\mathrm{H}), 7.09$ (br.s, $1 \mathrm{H}$, $\mathrm{NH}), 4.84\left(\mathrm{~d}, 1 \mathrm{H}, J_{\text {gem }}=11.7 \mathrm{~Hz}, 1-\mathrm{OC} \underline{\mathrm{HPh}}\right), 4.50$ $\left(\mathrm{d}, 1 \mathrm{H}, J_{\text {gem }}=11.7 \mathrm{~Hz}, 1-\mathrm{OC} \underline{\mathrm{HPh}}\right), 4.30(\mathrm{~d}, 1 \mathrm{H}$, $\left.J_{5^{\prime}, 5}=12.9 \mathrm{~Hz}, 5^{\prime}-\mathrm{H}\right), 4.16\left(\mathrm{~d}, 1 \mathrm{H}, J_{1,2}=7.3 \mathrm{~Hz}, 1-\right.$ $\mathrm{H}), 4.09\left(\mathrm{dd}, 1 \mathrm{H}, J_{5,5^{\prime}}=13.0 \mathrm{~Hz}, J_{5,4}=3.2 \mathrm{~Hz}, 5-\right.$ $\mathrm{H}), 3.98\left(\mathrm{t}, 2 \mathrm{H}, J_{\mathrm{NH}-\mathrm{CH} 2}=5.3 \mathrm{~Hz}, 6-\mathrm{H}\right), 3.80(\mathrm{~d}$, $\left.1 \mathrm{H}, J_{2,1}=7.2 \mathrm{~Hz}, 2-\mathrm{H}\right), 3.72\left(\mathrm{~s}, 3 \mathrm{H}, \mathrm{OCH}_{3}\right), 3.01$ (br.s, $1 \mathrm{H}, \mathrm{OH}), 2.24$ (d, $\left.1 \mathrm{H}, J_{3,4}=9.4 \mathrm{~Hz}, 3-\mathrm{H}\right)$, $2.11\left(\mathrm{dd}, 1 \mathrm{H}, J_{4,3}=9.4 \mathrm{~Hz}, J_{4,5}=3.0 \mathrm{z}, 4-\mathrm{H}\right) .{ }^{13} \mathrm{C}$ NMR $\left(100 \mathrm{MHz}, \mathrm{CDCl}_{3}\right): \delta=169.4\left(\mathrm{COOCH}_{3}\right)$, $165.0(\underline{\mathrm{CONH}}), 136.7-127.9(\mathrm{Ar}-\mathrm{C}), 115.9(\mathrm{CN})$, $101.9(\mathrm{C}-1), 70.3\left(\mathrm{O}_{-} H_{2} \mathrm{Ph}\right), 65.9$ (C-2), 62.0 (C5), $52.4\left(\mathrm{OCH}_{3}\right), 41.8\left(\underline{\mathrm{CH}}_{2} \mathrm{NH}\right), 32.3(\mathrm{C}-3), 27.4$ (C-4), 22.5 (C-6).

$$
\begin{array}{cccc}
\mathrm{C}_{18} \mathrm{H}_{20} \mathrm{~N}_{2} \mathrm{O}_{6} & (360.36) \\
\text { Calcd } & \text { C 59.99 } & \text { H } 5.59 & \text { N 7.77\%, } \\
\text { Found } & \text { C } 59.86 & \text { H } 6.06 & \text { N 7.47\%. }
\end{array}
$$

Benzyl 3,4-(C-cyano-benzyl glycinato)methano3,4-dideoxy- $\alpha$-D-arabinopyranoside (8): $0.5 \mathrm{~g}(1.73$ $\mathrm{mmol})$ of $\mathbf{5}$ and $0.4 \mathrm{~g}(1.98 \mathrm{mmol})$ of the hydrochloride of benzyl glycinate are taken up in $20 \mathrm{ml}$ DMF, cooled at $0{ }^{\circ} \mathrm{C}$ for $20 \mathrm{~min}$ and then $0.35 \mathrm{ml}$ (3.18 mmol) 4-methylmorpholine and $1.52 \mathrm{~g} \mathrm{(3.43}$ $\mathrm{mmol}) \mathrm{BOP}$ are added. The reaction is carried out and worked up in the same manner as described for 7. The purification is done by column chromatography over silica gel using dichloromethaneacetone $(9: 1)$ as eluent. Crystallization from ethyl acetate $/ n$-hexane provided white flakes of $\mathbf{8}$. Yield 
$0.5 \mathrm{~g}(66 \%)$, m.p. $140-142^{\circ} \mathrm{C},[\alpha]_{\mathrm{D}}=+1.7^{\circ}(\mathrm{c}=1$, $\left.\mathrm{CHCl}_{3}\right)$. - FD-MS: $m / z=437(\mathrm{M}+1)^{+} .-{ }^{1} \mathrm{H}$ NMR $\left(400 \mathrm{MHz}, \mathrm{CDCl}_{3}\right): \delta=7.35-7.24(\mathrm{~m}, 10 \mathrm{H}, \mathrm{Ar}-$ $\mathrm{H}$ ), 7.07 (br.t, $1 \mathrm{H}, J_{\mathrm{NH}-\mathrm{CH} 2}=5.3 \mathrm{~Hz}, \mathrm{CON} \underline{\mathrm{H}}$ ), $5.16\left(\mathrm{~s}, 2 \mathrm{H}, \mathrm{COOCH}{ }_{2} \mathrm{Ph}\right), 4.86(\mathrm{~d}, 1 \mathrm{H}$, gem $=11.7$ $\mathrm{Hz}, 1-\mathrm{OC} \underline{\mathrm{HPh}}), 4.51\left(\mathrm{~d}, 1 \mathrm{H}, J_{\mathrm{gem}}=11.7 \mathrm{~Hz}, 1-\right.$ OC $\underline{H P h}), 4.31\left(\mathrm{~d}, 1 \mathrm{H}, J_{5^{\prime}, 5}=12.9 \mathrm{~Hz}, 5^{\prime}-\mathrm{H}\right), 4.17$ $\left(\mathrm{d}, 1 \mathrm{H}, J_{1,2}=7.3 \mathrm{~Hz}, 1-\mathrm{H}\right), 4.12\left(\mathrm{dd}, 1 \mathrm{H}, J_{5,5^{\prime}}=\right.$ $\left.12.9 \mathrm{~Hz}, J_{5,4}=3.2 \mathrm{~Hz}, 5-\mathrm{H}\right), 4.03\left(\mathrm{dd}, 2 \mathrm{H}, J_{\mathrm{gem}}=\right.$ $\left.7.9 \mathrm{~Hz}, J_{\mathrm{CH} 2-\mathrm{NH}}=5.4 \mathrm{~Hz}, \mathrm{NHCH}_{2}\right), 3.82(\mathrm{dd}, 1 \mathrm{H}$, $\left.J_{2,1}=7.3 \mathrm{~Hz}, J_{2, \mathrm{OH}}=2.4 \mathrm{~Hz}, 2-\mathrm{H}\right), 2.59(\mathrm{~d}, 1 \mathrm{H}$, $\left.J_{\mathrm{OH}, 2}=3.3 \mathrm{~Hz}, \mathrm{OH}\right), 2.26\left(\mathrm{dd}, 1 \mathrm{H}, J_{3,2}=0.9 \mathrm{~Hz}\right.$, $\left.J_{3,4}=9.4 \mathrm{~Hz}, 3-\mathrm{H}\right), 2.18\left(\mathrm{dd}, 1 \mathrm{H}, J_{4,3}=9.4 \mathrm{~Hz}\right.$, $\left.J_{4,5}=3.0 \mathrm{~Hz}, 4-\mathrm{H}\right) . \quad-{ }^{13} \mathrm{C}$ NMR $(100 \mathrm{MHz}$,

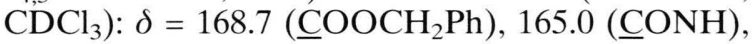
136.8-128.0 ( $\mathrm{Ar}-\mathrm{C}), 115.8(\mathrm{CN}), 101.9(\mathrm{C}-1), 70.4$ $\left(1-\mathrm{OCH}_{2} \mathrm{Ph}\right), 67.4\left(\mathrm{PhCH}_{2} \mathrm{O}\right), 66.1$ (C-2), 62.1 $(\mathrm{C}-5), 42.1\left(\mathrm{CH}_{2} \mathrm{NH}\right), 32.2(\mathrm{C}-3), 27.4(\mathrm{C}-4), 22.6$ (C-6).

\section{$\mathrm{C}_{24} \mathrm{H}_{24} \mathrm{~N}_{2} \mathrm{O}_{6}(436.45)$ \\ Calcd C 66.04 H 5.54 N 6.42\%, \\ Found C 65.49 H 5.85 N 6.14\%.}

Benzyl 3,4-(C-cyano-benzyl alaninato)methano3,4-dideoxy- $\alpha$ - $D$-arabinopyranoside (9): Is prepared from 5 by the same procedure as that applied for 8 using $0.4 \mathrm{~g}(1.85 \mathrm{mmol})$ of the hydrochloride of benzyl alaninate. The product, after purification through column chromatography, crystallized from ethyl acetate $/ n$-hexane as colourless needles. Yield $0.55 \mathrm{~g}(71 \%)$, m.p. $118^{\circ} \mathrm{C}$, $[\alpha]_{\mathrm{D}}=+1.7^{\circ}\left(\mathrm{c}=1, \mathrm{CHCl}_{3}\right)$. - FD-MS: $\mathrm{m} / \mathrm{z} 451$ $(\mathrm{M}+1)^{+} .-{ }^{1} \mathrm{H}$ NMR $\left(400 \mathrm{MHz}, \mathrm{CDCl}_{3}\right): \delta=7.37-$ $7.31(\mathrm{~m}, 10 \mathrm{H}, \mathrm{Ar}-\mathrm{H}), 6.93\left(\mathrm{~d}, 1 \mathrm{H}, J_{\mathrm{NH}}=7.2 \mathrm{~Hz}\right.$, $\mathrm{NH}), 5.19\left(\mathrm{~d}, 1 \mathrm{H}, J_{\mathrm{gem}}=12.2 \mathrm{~Hz}, \mathrm{COOC} \underline{\mathrm{HPh}}\right)$, $5.14\left(\mathrm{~d}, 1 \mathrm{H}, J_{\text {gem }}=12.2 \mathrm{~Hz}, \mathrm{COOC} \underline{\mathrm{HPh}}\right), 4.87(\mathrm{~d}$, $\left.1 \mathrm{H}, J_{\text {gem }}=11.6 \mathrm{~Hz}, 1-\mathrm{OC} \underline{\mathrm{HPh}}\right), 4.55(\mathrm{q}, 1 \mathrm{H}$, $\left.J_{\mathrm{CH}, \mathrm{CH} 3}=7.2 \mathrm{~Hz}\right), 4.51\left(\mathrm{~d}, 1 \mathrm{H}, J_{\mathrm{gem}}=11.6 \mathrm{~Hz}, 1-\right.$

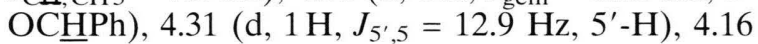
$\left(\mathrm{d}, 1 \mathrm{H}, J_{1,2}=7.3 \mathrm{~Hz}, 1-\mathrm{H}\right), 4.11\left(\mathrm{dd}, 1 \mathrm{H}, J_{5,5^{\prime}}=\right.$ $\left.12.9 \mathrm{~Hz}, J_{5,4}=3.3 \mathrm{~Hz}, 5-\mathrm{H}\right), 3.83\left(\mathrm{ddd}, 1 \mathrm{H}, J_{2,1}=\right.$ $\left.7.3 \mathrm{~Hz}, J_{2,3}=1.0 \mathrm{~Hz}, J_{2, \mathrm{OH}}=3.0 \mathrm{~Hz}, 2-\mathrm{H}\right), 2.34(\mathrm{~d}$, $1 \mathrm{H}, J_{\mathrm{OH}, 2}=3.0 \mathrm{~Hz}, \mathrm{OH}, 2.27\left(\mathrm{dd}, 1 \mathrm{H}, J_{3,4}=9.4\right.$ $\left.\mathrm{Hz}, J_{3,2}=1.0 \mathrm{~Hz}, 3-\mathrm{H}\right), 2.06\left(\mathrm{dd}, 1 \mathrm{H}, J_{4,3}=9.3 \mathrm{~Hz}\right.$, $\left.J_{4,5}=3.0 \mathrm{~Hz}, 4-\mathrm{H}\right), 1.44\left(\mathrm{~d}, 3 \mathrm{H}, J_{\mathrm{CH} 3, \mathrm{CH}}=7.2 \mathrm{~Hz}\right.$, $\left.\mathrm{CH}_{3}\right) .-{ }^{13} \mathrm{C}$ NMR $\left(100 \mathrm{MHz}, \mathrm{CDCl}_{3}\right): \delta=171.7$ $\left(\mathrm{CH}\left(\mathrm{CH}_{3}\right) \underline{\mathrm{COO}}\right), \quad 164.1 \quad(\underline{\mathrm{CONH}}), \quad 136.5-128.1$ $(\mathrm{Ar}-\mathrm{C}), \quad 115.8 \quad(\mathrm{CN}), \quad 101.9 \quad(\mathrm{C}-1), \quad 70.3 \quad$ (1$\left.\mathrm{OCH}_{2} \mathrm{Ph}\right), 67.3\left(\mathrm{PhCH}_{2} \mathrm{OCO}\right), 66.1$ (C-12), 62.1 $(\mathrm{C}-5), 49.2\left[\underline{\mathrm{CH}}\left(\mathrm{CH}_{3}\right)\right], 31.9(\mathrm{C}-3), 27.3(\mathrm{C}-4), 18.0$ (C-6), $17.9\left(\mathrm{CH}_{3}\right)$.

$\mathrm{C}_{25} \mathrm{H}_{26} \mathrm{~N}_{2} \mathrm{O}_{6}(450.47)$

Calcd C 66.65 H 5.82 N 6.22\%,

Found C 66.72 H 5.68 N $6.35 \%$.
Benzyl 3,4-(C-cyano-benzyl glycinato)methano3,4-dideoxy- $\beta$-L-arabinopyranoside (10): The synthesis of $\mathbf{1 0}$ is performed in an analogous manner as that of $8.0 .65 \mathrm{~g}(2.18 \mathrm{mmol}) \mathbf{6}$ are treated with $0.5 \mathrm{~g}(2.47 \mathrm{mmol})$ benzyl glycinate hydrochloride in the presence of $0.5 \mathrm{ml}(4.48 \mathrm{mmol})$ 4-methylmorpholine and $2 \mathrm{~g}(4.51 \mathrm{mmol}) \mathrm{BOP}$. Yield $0.7 \mathrm{~g}$ $(71 \%)$, m.p. $112^{\circ} \mathrm{C},[\alpha]_{\mathrm{D}}=+9.9^{\circ}\left(\mathrm{c}=1, \mathrm{CHCl}_{3}\right)$. FD-MS: $m / \mathrm{z}=437(\mathrm{M}+1)^{+} .-1 \mathrm{H}$ NMR $(400 \mathrm{MHz}$, $\left.\mathrm{CDCl}_{3}\right): \delta=7.38-7.30(\mathrm{~m}, 10 \mathrm{H}, \mathrm{Ar}-\mathrm{H}), 6.98(\mathrm{t}$, $\left.1 \mathrm{H}, \quad J_{\mathrm{NH}}=5.3 \mathrm{~Hz}, \mathrm{CON \underline {H }}\right), 5.17(\mathrm{~s}, 2 \mathrm{H}$, $\left.\mathrm{COOC}_{2} \mathrm{Ph}\right), 4.84\left(\mathrm{~d}, 1 \mathrm{H}, J_{1,2}=4.6 \mathrm{~Hz}, 1-\mathrm{H}\right), 4.75$ $\left(\mathrm{d}, 1 \mathrm{H}, J_{\text {gem }}=11.6 \mathrm{~Hz}, 1-\mathrm{OC} \underline{\mathrm{HPh}}\right), 4.53(\mathrm{~d}, 1 \mathrm{H}$, $J_{\text {gem }}=11.6 \mathrm{~Hz}, 1-\mathrm{OC} \underline{\mathrm{HPh}}$ ), 4.13-3.99 (overlapping multiplets, $\left.3, \mathrm{NHC}^{-} \underline{\mathrm{H}}_{2}+5^{\prime}-\mathrm{H}\right), 3.86\left(\mathrm{~d}, 1 \mathrm{H}, J_{5,5^{\prime}}=\right.$ $12.9 \mathrm{~Hz}, 5-\mathrm{H}), 3.82\left(\mathrm{dd}, 1 \mathrm{H}, J_{2,1} 4.6 \mathrm{~Hz}, J_{2,3}=1.2\right.$ $\mathrm{Hz}, 2-\mathrm{H}), 2.83\left(\mathrm{~d}, 1 \mathrm{H}, J_{\mathrm{OH}}=11.1 \mathrm{~Hz}, \mathrm{OH}\right), 2.23$ $\left(\mathrm{d}, 1 \mathrm{H}, J_{3,4}=9.9 \mathrm{~Hz}, 3-\mathrm{H}\right), 2.18\left(\mathrm{dd}, 1 \mathrm{H}, J_{4,3} 9.9\right.$ $\left.\mathrm{Hz}, J_{4,5^{\prime}}=3.5 \mathrm{~Hz}, 4-\mathrm{H}\right) .-{ }^{13} \mathrm{C}$ NMR $(100 \mathrm{MHz}$, $\left.\mathrm{CDCl}_{3}\right): \delta=168.7\left(\underline{\mathrm{COOC}}_{2} \mathrm{Ph}\right), 165.0(\underline{\mathrm{CONH}})$, 136.33-128.1 (Ar-C), 93.17 (C-1), 70.24 (1$\left.\mathrm{OCH}_{2} \mathrm{Ph}\right), 67.39\left(\mathrm{PHCH}_{2} \mathrm{OCO}\right), 62.3$ (C-2), 54.1 $(\widetilde{\mathrm{C}}-5), 42.0\left(\mathrm{NH}^{\mathrm{C}} \mathrm{H}_{2} \mathrm{CO}\right), 30.9$ (C-3), 26.8 (C-4), 22.8 (C-6).

\section{$\mathrm{C}_{24} \mathrm{H}_{24} \mathrm{~N}_{2} \mathrm{O}_{6}(436.45)$ \\ Calcd C 66.04 H 5.54 N 6.42\%, \\ Found C 65.27 H 5.68 N 6.19\%.}

Benzyl 3,4-[(C-cyano-amido)methano-(benzyl3,4-("exo"-methylene)-methano-3,4-dideoxy- $\beta$ - $L$ arabinopyranosido)]methano-3,4-dideoxy- $\beta$ - $L$ arabinopyranoside (12): $0.60 \mathrm{~g}(2.07 \mathrm{mmol}) 6$ and $0.52 \mathrm{~g}(2.08 \mathrm{mmol})$ of $\mathbf{1 1}$ are dissolved in $20 \mathrm{ml}$ dimethylformamide and then $0.45 \mathrm{ml}(4.09 \mathrm{mmol})$ 4-methylmorpholine and $1.83 \mathrm{~g}(4.13 \mathrm{mmol}) \mathrm{BOP}$ are added. The reaction product is worked up and purified as described for $\mathbf{7}$ to furnish, after crystallization from ethyl acetate $/ n$-hexane, colourless needles. Yield $0.75 \mathrm{~g}(69.5 \%)$, m.p. $67-70{ }^{\circ} \mathrm{C}$, $[\alpha]_{\mathrm{D}}=+178.7^{\circ}\left(\mathrm{c}=1, \mathrm{CHCl}_{3}\right)$. FD-MS: $m / z=406$ $\left(\mathrm{M}-\mathrm{CN}-\mathrm{CH}_{2} \mathrm{Ph}\right)^{+} . \quad-{ }^{1} \mathrm{H}$ NMR $(400 \mathrm{MHz}$, $\left.\mathrm{CDCl}_{3}\right): \delta=7.38-7.30(\mathrm{~m}, 10 \mathrm{H}, \mathrm{Ar}-\mathrm{H}), 6.54$ (br.t, $\left.J_{\mathrm{NH}-\mathrm{CH} 2}=5.5 \mathrm{~Hz}, \mathrm{NH}\right), 4.85\left(\mathrm{~d}, 1 \mathrm{H}, J_{1,2}=4.7 \mathrm{~Hz}\right.$, $1-\mathrm{H}), 4.77\left(\mathrm{~d}, 1 \mathrm{H}, J_{\mathrm{gem}}=11.7 \mathrm{~Hz}, 1-\mathrm{OC} \underline{\mathrm{HPh}}\right), 4.76$ $\left(\mathrm{d}, 1 \mathrm{H}, J_{\text {gem }}=11.6 \mathrm{~Hz}, 1-\mathrm{OC} \underline{\mathrm{HPh}}\right), 4.55(\mathrm{~d}, 1 \mathrm{H}$, $\left.J_{1^{\prime}, 2^{\prime}}=3.4 \mathrm{~Hz}, 1^{\prime}-\mathrm{H}\right), 4.54\left(\mathrm{~d}, \overline{1 \mathrm{H}}, J_{\mathrm{gem}}=11.8 \mathrm{~Hz}\right.$, 1-OCHPh), 4.49 (d, $\left.1 \mathrm{H}, J_{\text {gem }} 11.7 \mathrm{~Hz}, 1-\mathrm{OC} \underline{\mathrm{HPh}}\right)$, 4.09-4.05 (overlapping multiplets, $2 \mathrm{H}, 5^{\prime \prime}-\mathrm{H}+5^{\prime \prime \prime}-$ $\mathrm{H}), 3.90\left(\mathrm{~d}, 1 \mathrm{H}, J_{5.5^{\prime \prime}}=12.7 \mathrm{~Hz}, 5-\mathrm{H}\right), 3.83(\mathrm{ddd}$, $1 \mathrm{H}, J_{2,1}=4.7 \mathrm{~Hz}, J_{2,3}=1.0 \mathrm{~Hz}, J_{2, \mathrm{OH}}=11.2 \mathrm{~Hz}$, $2-\mathrm{H}), 3,78\left(\mathrm{ddd}, 1 \mathrm{H}, J_{2^{\prime}, 1^{\prime}}=4.5 \mathrm{~Hz}, J_{2^{\prime}, 3^{\prime}}=1.1 \mathrm{~Hz}\right.$, $\left.J_{2^{\prime}, \mathrm{OH}}=9.0 \mathrm{~Hz}, 2^{\prime}-\mathrm{H}\right), 3.73\left(\mathrm{~d}, 1 \mathrm{H}, J_{5^{\prime}, 5}=11.3 \mathrm{~Hz}\right.$, $\left.5^{\prime}-\mathrm{H}\right), 3.23\left(\mathrm{~m}, 1 \mathrm{H}, J_{\text {vic }}=6.2 \mathrm{~Hz}, 7-\mathrm{H}\right), 3.13(\mathrm{~m}$, $\left.1 \mathrm{H}, J_{\text {vic }} 5.8 \mathrm{~Hz}, 7-\mathrm{H}\right), 2.75\left(\mathrm{~d}, 1 \mathrm{H}, J_{\mathrm{OH}, 2}=11.2\right.$ $\mathrm{Hz}, \mathrm{OH}), 2.61\left(\mathrm{~d}, 1 \mathrm{H}, J_{\mathrm{OH}, 2^{\prime}}=9.0 \mathrm{~Hz}, \mathrm{OH}\right), 2.22$ 
$\left(\mathrm{d}, 1 \mathrm{H}, J_{3,4}=9.9 \mathrm{~Hz}, 3-\mathrm{H}\right), 2.18\left(\mathrm{dd}, 1 \mathrm{H}, J_{4,3}=\right.$ $9.9 \mathrm{~Hz}, J_{4.5}=3.2 \mathrm{~Hz}, 4-\mathrm{H}$ ), $1.05-0.92$ (overlapping multiplets, $\left.3 \mathrm{H}, 33^{\prime}-\mathrm{H}, 4^{\prime}-\mathrm{H}+6^{\prime}-\mathrm{H}\right) . \quad-{ }^{13} \mathrm{C}$ NMR $\left(100 \mathrm{MHz}, \mathrm{CDCl}_{3}\right): \delta=164.5(\mathrm{CONH}), 136.9-$ 127.8 ( $\mathrm{Ar}-\mathrm{C}), 116.1(\mathrm{CN}), 93.8$ (C-1'), 93.2 (C-1), $70.2\left(1^{\prime}-\mathrm{OCH}_{2} \mathrm{Ph}\right), 69.7\left(1-\mathrm{OCH}_{2} \mathrm{Ph}\right), 64.5(\mathrm{C}-2)$, 62.4 (C-2'), 58.0 (C-5), $54.2\left({\left.\mathrm{C}-5^{\prime}\right),}^{\prime} 43.6\right.$ (C-7), 30.6 (C-3), 26.4 (C-3'), 22.8 (C-6), 20.5 (C-4), 20.3 $\left(\mathrm{C}-4^{\prime}\right), 15.0\left(\mathrm{C}-6^{\prime}\right)$.

$\mathrm{C}_{29} \mathrm{H}_{32} \mathrm{~N}_{2} \mathrm{O}_{7}(520.56)$

Calcd C 66.91 H 6.20 N 5.38\%,

Found C 66.88 H 6.51 N 4.64\%.

\section{Acknowledgement}

The authors are indebted to Ministerium für Wissenschaft und Kunst Baden-Württemberg and to German Academic Exchange Service for providing scholarships to F. Z. \& A.F. and a grant to A. M., respectively. Financial support by the Fonds der Chemischen Industrie is greatly acknowledged.
[1] A. Fatima, F. Zaman, M. S. Shekhani, A. Malik, W. Voelter, Liebigs Ann. Chem. 1990, 389.

[2] D. Buchanan, D. M. Clode, N. Vethaviyasar, J. Chem. Soc. Perkin Trans. 1, 1976, 1449.

[3] A. Holý, F. Šorm, Coll. Czech. Chem. Commun. 34, 3383 (1969).

[4] R. Kimmich, W. Voelter, Liebigs Ann. Chem. 1100 (1981).

[5] A. Fournier, C.-T. Wang, A. M. Felix, Int. J. Pept. Prot. Res. 31, 86 (1988).
[6] B. Castro, J. R. Dormoy, G. Evin, C. Selve, Tetrahedron Lett. 1975, 1219.

[7] U. Schöllkopf, B. Hupfelld, R. Gull, Angew. Chem. 98, 755 (1986).

[8] U. Schöllkopf, R. Harms, D. Hoppe, Liebigs. Ann. Chem. 1973, 611.

[9] E. Breitmaier, W. Voelter, Carbon-13 NMR Spectroscopy, 3rd Ed., VCH Verlagsgesellschaft $\mathrm{mbH}$, Weinheim (1987). 